\title{
A Study of the Impact of Lockdown on Vaccination Coverage at a Tertiary Care Center
}

\author{
Purvi Patel ${ }^{1}$, Halak Vasavada ${ }^{2}$, Snehal V Patel $^{3}$, Khushbu Patel $^{4}$, Payal Rathva ${ }^{5}$
}

\begin{abstract}
Aim and objective: The aim and objective of the this study is to study the impact of lockdown on vaccination coverage at a tertiary care center. Materials and methods: It was a retrospective analytical study carried out at Shardaben Hospital, Saraspur, Ahmedabad, Gujarat.. The study period was April-May 2019 and April-May 2020.

Results: In our study, during lockdown period, number of vaccinated children were lower in May (837) as compared to April $(1,352)$ in year 2020. Total number of deliveries increased to 507 in April 2020 compared to 388 in April 2019 resulting in more inborn vaccination in April 2020 (92.50\%). In May 2020, total number of deliveries (386) were less compared to April 2020 (507) and May 2019 (505). The birth vaccination is $92.50 \%$ in April 2020 and $83.56 \%$ in May 2020. In April, the DPT booster vaccination rates reduced by $87 \%$ while MR vaccination reduced by $58 \%$. In May, there was reduction in DPT booster at 5 years (96.66\%) and pentavalent third dose (78.94\%) and MR (78.57\%).

Conclusion: There has been significant reduction in attendance to the immunization clinic for routine vaccination during the lockdown period secondary to coronavirus pandemic. The reduction has been more in May 2020 as compared to April 2020 because of peaking of cases during that time in the area with it being declared as containment zone during that period.

Clinical significance: There has been a significant impact of lockdown on routine immunization under five children and an urgent vaccination drive is the need of the hour.

Keywords: Immunization, Lockdown, Pandemic, Vaccination.

Pediatric Infectious Disease (2020): 10.5005/jp-journals-10081-1264
\end{abstract}

\section{INTRODUCTION}

The world has been gripped by the COVID-19 pandemic, which has been declared as a global health emergency by the WHO on March $11,2020 .^{1}$ The disease itself as well as its management in the form of nationwide lockdown has had a far-reaching impact on various aspects of public health.

An estimated 20-22 lakh children $<1$ year in India are targeted for vaccination under national programs every month, according to official sources. ${ }^{2}$ The WHO and UNICEF estimates of immunization coverage 2018 revision shows an estimated 92\% immunization coverage in 2018 in India. ${ }^{3}$

The hampering of routine immunization in children against vaccine-preventable diseases (VPDs) like diphtheria, pertussis, tetanus, measles, and polio during the 2 months coronavirus lockdown has triggered concern about possible resurgence of VPDs.

It is feared that lockdown may undo the benefit of wide immunization coverage achieved by India in the past few years and also nullify the benefit of the measles-rubella campaign of 2018 and jeopardize the polio-free status accorded to India by the WHO. ${ }^{4}$

Immunization in most remote parts of India is carried out by ASHA workers who work under the Ministry of Health and Family Welfare. The ASHA workers have been given many tasks related to COVID-19 pandemic like conducting door-to-door surveys, creating awareness campaigns, and so on. Hence, these workers have not been able to provide outreach immunization services. ${ }^{5}$

Though birth doses of OPV, hepatitis B, and BCG have been given at birth centers, the subsequent doses can often be missed due to inaccessibility of health care due to lack of public transport, fear of COVID-19 infection on visiting hospitals, and many other factors directly or indirectly due to lockdown. Many states of India like UP, Bihar, Kerala, Karnataka, and Rajasthan stopped
${ }^{1-5}$ Department of Pediatrics, Smt NHL Municipal Medical College, Ahmedabad, Gujarat, India

Corresponding Author: Purvi Patel, Department of Pediatrics, Smt NHL Municipal Medical College, Ahmedabad, Gujarat, India, Phone: +91 9825661647, e-mail: evergreen.purvi@yahoo.in

How to cite this article: Patel P, Vasavada H, Patel SV, et al. A Study of the Impact of Lockdown on Vaccination Coverage at a Tertiary Care Center. Pediatr Inf Dis 2020;2(2):51-54.

Source of support: Nil

Conflict of interest: None

immunization activities for a short period of time, though the activity continued uninterrupted in Gujarat. ${ }^{6}$

Third, supply chain of vaccines might be affected due to exportimport restrictions and intrastate and interstate travel restrictions.

This difficult situation calls for innovative ways to face these challenges and avert the disaster of VPD epidemics in the postCOVID era.

Data related to impact of lockdown on immunization coverage in India are scarce and no official statistics are as yet available. This study aims to study the fallout of lockdown with respect to immunization visits in children.

\section{Materials and Methods}

It was a retrospective analytical study carried out at Shardaben Hospital, Saraspur, Ahmedabad, Gujarat.

It is a tertiary care center attached to a municipal medical college. It is located in a containment area-i.e., high transmission zone for SARS-CoV2 infections. It is a center that catered to non- 
COVID patients during the pandemic, with suspected patients transferred to COVID hospitals when they tested positive.

The study period was April 1, 2020, to May 31, 2019, and AprilMay 2020. The area was under lockdown during this period. It was a containment zone with strict lockdown between May 3, 2020, and May 31, 2020. All children attending immunization clinic aged 0 days to 5 years in April and May 2019 and April and May 2020 were included in the study. Immunization data of these children were analyzed from the hospital records along with their demography and results were tabulated. Comparative study was done between the two sets of data and appropriate statistical tests applied.

The National Immunization Schedule was followed for vaccination. $^{7}$

\section{Results}

The present study shows that during the lockdown period the absolute numbers of all vaccines administered were lower in May (837) as compared to April $(1,352)$ in the year 2020. This can be attributed to stricter lockdown policies by the government in May as compared to April. Lack of public transport and fear of contracting COVID-19 infection on hospital visit might be the reason for this decline. The area where our hospital is located was under the containment zone from May 3, 2020 till May 31, 2020, so total number of deliveries also reduced (Table 1).

So in our study, total number of deliveries increased in April 2020 compared to April 2019, resulting in more inborn vaccination in April 2020 (Table 2).

The Chi-square statistic is 9.8353. The $p$ value is 0.007316 . The result is significant as $p<0.05$. So, there is significant increase in vaccination in April 2020.

Similarly, for the month of May 2019 and May 2020, the Chisquare statistic is 6.9659 . The $p$ value is 0.0030717 . The result is significant at $p<0.05$. So, there is significant decrease in vaccination in May 2020.

Table 1: Vaccination during lockdown period

\begin{tabular}{lcc}
\hline Vaccines & April 2020 & May 2020 \\
\hline BCG & 469 & 307 \\
OPV & 469 & 307 \\
HEP B & 362 & 186 \\
PENTA 1 & 28 & 24 \\
PENTA 2 & 12 & 5 \\
PENTA 3 & 6 & 4 \\
MR & 5 & 3 \\
DPT & 1 & 1 \\
Total & 1,352 & 837 \\
\hline
\end{tabular}

Table 2: Total deliveries vs birth vaccination

\begin{tabular}{llll}
\hline & Total deliveries & $\begin{array}{l}\text { Birth } \\
\text { vaccination }\end{array}$ & $\begin{array}{l}\text { Vaccination } \\
\text { percentage }\end{array}$ \\
\hline April 2019 & 388 & 289 & 74.48 \\
April 2020 & 507 & 469 & 92.50 \\
\hline & & Birth & $\begin{array}{l}\text { Vaccination } \\
\text { percentage }\end{array}$ \\
\hline May 2019 & 505 & 422 & 83.56 \\
May 2020 & 386 & 307 & 79.53 \\
\hline
\end{tabular}

The Chi-square statistic is 2.9391 . The $p$ value is 0.568063 (significant value $p<0.05$ ). So, statistically, there is no significant difference in vaccination between April 2019 and April 2020 (Table 3).

For the month of May 2019 and May 2020, the Chi-square statistic is 10.7778 . The $p$ value is 0.029178 . The result is significant at $p<0.05$. So, there is significant decrease in vaccination in May 2020 (Figs 1 and 2).

Overall, vaccination coverage was less during the whole lockdown period compared to last year except birth vaccination increased in April 2020 due to more number of deliveries in municipal hospital (Table 4).

\section{Vaccination Status and Comparison}

From above data, we can say that in April 2019 15.91\% babies came for first dose of pentavalent vaccination after birth dose, which fall to $5.91 \%$ in April 2020 (Table 5).

In May 2019, 12.79\% babies came for first dose of pentavalent after birth dose, which fall to $7.81 \%$ in May 2020 (Table 6).

\section{Discussion}

As attendance in well baby and immunization clinics dropped drastically for the fear of exposure to coronavirus, public health experts fear that another health crisis may be looming in the near future. The incidence of VPDs is expected to rise as a collateral damage due to strict lockdown policies and circumstances surrounding coronavirus spread. ${ }^{8}$

The present study shows that during the lockdown period the absolute numbers of all vaccines administered were lower in May (837) as compared to April $(1,352)$ in the year 2020.

Comparing the number of deliveries in the hospital during April and May 2019 and 2020, and accordingly the number of birth doses of BCG given to the neonates, it can be said that the total number

Table 3: Vaccination comparison between 2019 and 2020 (April, May)

\begin{tabular}{lll}
\hline Vaccines & April 2019 & April 2020 \\
\hline BCG & 289 & 469 \\
OPV & 395 & 469 \\
HEP B 0 dose & 251 & 362 \\
\hline Vaccines & May 2019 & May 2020 \\
\hline BCG & 422 & 307 \\
OPV & 554 & 307 \\
HEP B 0 dose & 300 & 186 \\
\hline Vaccines & April 2019 & April 2020 \\
\hline PENTA 1 & 46 & 28 \\
PENTA 2 & 26 & 12 \\
PENTA 3 & 10 & 6 \\
MR & 12 & 5 \\
DPT & 8 & 1 \\
\hline Vaccines & 14 & 3 \\
\hline PENTA 1 & 30 & 24 \\
PENTA 2 & May 2019 & 5 \\
PENTA 3 & 54 & May 2020 \\
MR & 22 & 24 \\
DPT & 19 & 3 \\
\hline & 14 & 3 \\
\hline
\end{tabular}




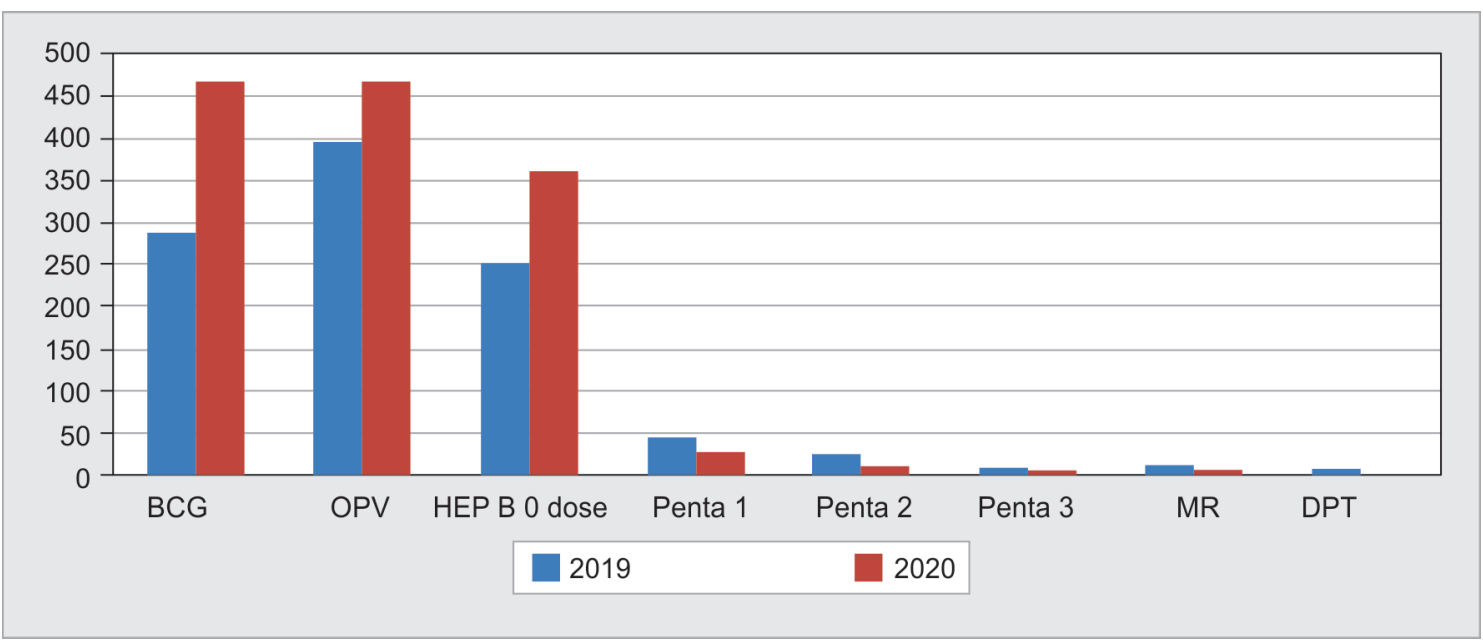

Fig. 1: Comparison of vaccination status between April 2019 and April 2020

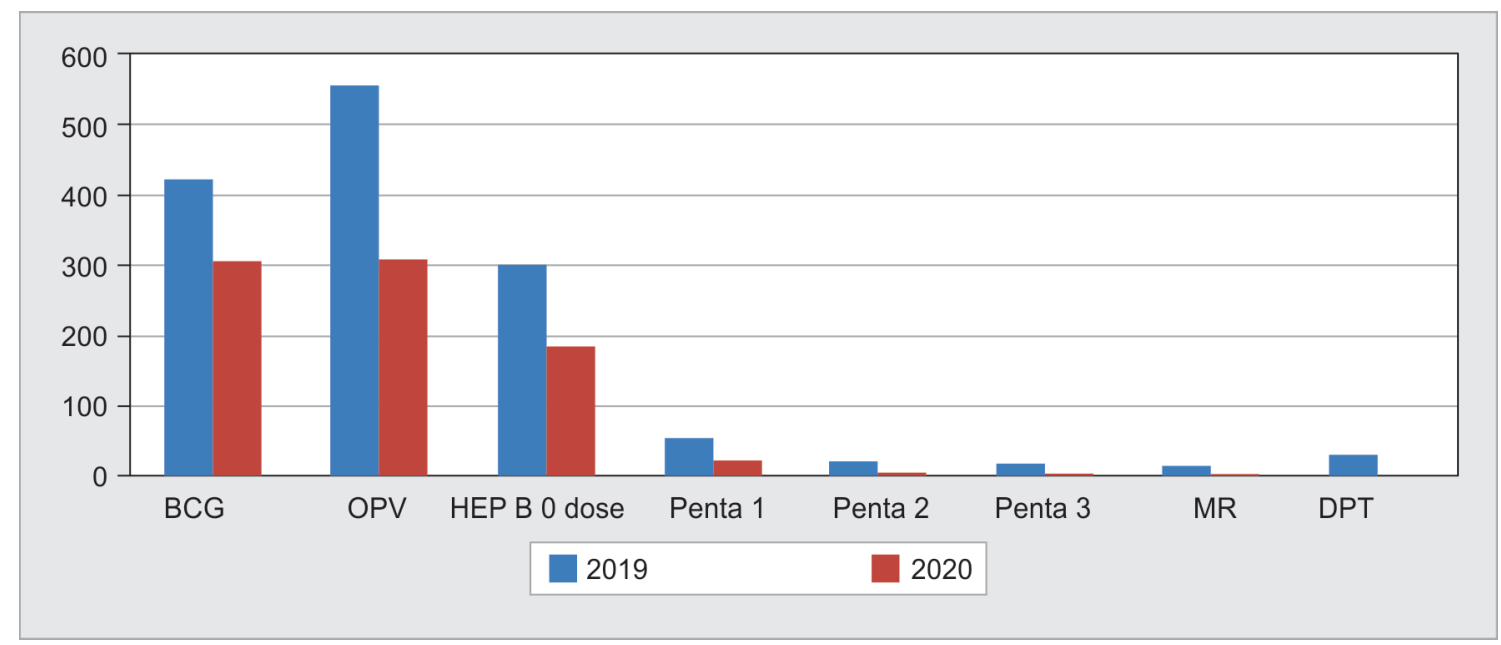

Fig. 2: Comparison of vaccination status between May 2019 and May 2020

Table 4: Vaccination trend in lockdown

\begin{tabular}{llcl}
\hline Vaccines & April 2019 & April 2020 & Difference \\
\hline BCG & 289 & 469 & $38.37 \%(\uparrow)$ \\
OPV & 395 & 469 & $15.77 \%(\uparrow)$ \\
HEP B 0 dose & 251 & 362 & $30.66 \%(\uparrow)$ \\
PENTA 1 & 46 & 28 & $39.13 \%(\downarrow)$ \\
PENTA 2 & 26 & 12 & $53.84 \%(\downarrow)$ \\
PENTA 3 & 10 & 6 & $40.00 \%(\downarrow)$ \\
MR & 12 & 5 & $58.33 \%(\downarrow)$ \\
DPT & 8 & 1 & $87.50 \%(\downarrow)$ \\
\hline Vaccines & May 2019 & May 2020 & Difference \\
\hline BCG & 422 & 307 & $27.25 \%(\downarrow)$ \\
OPV & 554 & 307 & $44.58 \%(\downarrow)$ \\
HEP B 0 dose & 300 & 186 & $38.00 \%(\downarrow)$ \\
PENTA 1 & 54 & 24 & $55.55 \%(\downarrow)$ \\
PENTA 2 & 22 & 5 & $77.27 \%(\downarrow)$ \\
PENTA 3 & 19 & 4 & $78.94 \%(\downarrow)$ \\
MR & 14 & 3 & $78.57 \%(\downarrow)$ \\
DPT & 30 & 1 & $96.66 \%(\downarrow)$ \\
\hline
\end{tabular}

Table 5: Birth dose of BCG vs first dose of pentavalent

\begin{tabular}{llc}
\hline Vaccines & April 2019 & April 2020 \\
\hline Birth dose (BCG) & 289 & 469 \\
PENTA 1 & 46 & 28 \\
& $15.91 \%$ & $5.97 \%$ \\
\hline Vaccines & May 2019 & May 2020 \\
\hline Birth dose (BCG) & 422 & 307 \\
PENTA 1 & 54 & 24 \\
& $12.79 \%$ & $7.81 \%$ \\
\hline
\end{tabular}

Table 6: Antenatal vaccination

\begin{tabular}{lllll}
\hline & April 2019 & April 2020 & May 2019 & May 2020 \\
\hline TT-1 & 70 & 190 & 94 & 26 \\
TT-2 & 59 & 84 & 74 & 17 \\
\hline
\end{tabular}

of deliveries increased in April 2020 (507) as compared to April 2019 (469). This may be a natural progression due to increase in birth rate over the period of 1 year or it may be due to the fact that many private nursing homes were shut down during the initial period of 
lockdown and patients had to avail the health care in urban health centers and teaching hospitals during that time.

In May 2020, total number of deliveries (386) were less compared to April 2020 (507) and May 2019 (505).

The birth vaccination percentage also follows the same trend as the number of deliveries as most of these neonates are given birth vaccination while the mother has been admitted for delivery. Due to less options available, the vaccination percentage rose to $92.50 \%$ in April 2020 as compared to $74.48 \%$ in April 2019. Also, the percentage fell from $83.56 \%$ in May 2019 to $79.53 \%$ in May 2020.

Comparing the statistical data for individual vaccines, as regards the birth vaccines, for $B C G, O P V$, and hepatitis $B 0$ dose, the Chi-square statistic is 9.8353 . The $p$ value is 0.007316 . The result is significant at $p<0.05$. So, there is significant increase in vaccination in April 2020. This follows the general trend of increase in number of deliveries in April 2020 compared to April 2019 as described above.

Similarly, for the month of May 2019 and May 2020, the chisquare statistic is 6.9659 . The $p$ value is 0.0030717 . The result is significant at $p<0.05$. So, there is significant decrease in vaccination in May 2020.

Although the absolute numbers in vaccination for pentavalent vaccine, MR vaccine, and DPT boosters have reduced during April 2020 as compared to April 2019, the Chi-square statistic is 2.9391. The $p$ value is 0.568063 (significant value $p<0.05$ ). So, statistically, there is no significant difference in vaccination between April 2019 and April 2020. The impact of coronavirus outbreak on vaccination and hence public health has been less in April 2020.

For the month of May 2019 and May 2020, the Chi-square statistic is 10.7778 . The $p$ value is 0.029178 . The result is significant at $p<0.05$. So, there is significant decrease in vaccination in May 2020. The impact is now starkly apparent with marked reduction in vaccine coverage.

Overall vaccination coverage was less during the whole lockdown period compared to last year except birth vaccination increased in April 2020 due to more number of deliveries in municipal hospital.

In April, the DPT booster vaccination rates reduced by more than $87 \%$ while MR vaccination reduced by $58 \%$.

In May, the major setback has been for the DPT booster at 5 years $(96.66 \%$ reduction) followed by pentavalent third dose (78.94\%) and MR (78.57\%).

The above data show that the impact of lockdown on vaccination coverage has been significant, more in the month of May 2020 as compared to April 2020. Also, the later doses including MR and DPT booster were affected more as compared to birth vaccines and primary doses of vaccination.

The area where the hospital is located was under strict lockdown and became a containment zone from May 3, 2020, to May 31, 2020, as cases of COVID-19 peaked in the area during that time. Hence, the impact has been more in May as compared to April. Lack of public transport and fear of contracting COVID-19 infection on hospital visit might be the reason for this decline.

Data from Indian hospitals regarding reduction in vaccination coverage with percentage reductions are not yet available; however, in the United States also, data collected from 1,000 independent pediatricians showed $50 \%$ reduction in measles and rubella vaccination during the first 2 weeks of April, diphtheria and whooping cough by $42 \%$. Massachusetts and Minnesota reported a significant $68 \%$ and $71 \%$ drop in immunization during this period. ${ }^{9}$
It is a known fact that reduction or delay in vaccination can lead to far-reaching consequences for public health and experts fear outbreak of VPDs including measles in the near future. ${ }^{10}$

Fortunately, there has been no interruption in supply chain of vaccines throughout the lockdown period and vaccines were freely available for all those who presented for the same. Also, being a non-COVID center and due to the policies of Government of Gujarat, even during the strictest lockdown, the immunization services were not interrupted even for a single day and with team approach and proper IPC measures, healthcare workers could cope with vaccination during the difficult period, though outreach services could have been affected.

\section{Conclusion}

There has been a significant reduction in attendance to the immunization clinic for routine vaccination during the lockdown period secondary to coronavirus pandemic as compared to previous year. The maximum reduction has been for MR vaccine and DPT booster followed by pentavalent vaccines. Birth dose has been relatively less affected.

The reduction has been more in May 2020 as compared to April 2020 because of peaking of cases during that time in the area with it being declared as the containment zone during that period. Logistic difficulties in reaching the hospital and fear of exposure to the coronavirus may have been the main factors for the reduction.

Studies are needed to evaluate a rise in cases of VPDs including measles in the near future are needed to study the adverse outcome of lockdown on child health.

The need of the hour is to find innovative ways to remedy this. With unlock-1, a special vaccination drive to cover up for all the vaccination lapses in the community is must, else we might land up into epidemics of VPDs in near future. There is also a need to intensify outreach services of ASHA workers especially for immunization. It has to be done now and not leave it for future.

\section{Clinical Significance}

There has been a significant impact of lockdown on routine immunization in under 5 children and an urgent vaccination drive is the need of the hour.

\section{References}

1. World Health Organization Timeline COVID 19, https://www.who.int.

2. Vaccination and Immunization Statistics-UNICEF data. https://data. unicef.org.

3. India: WHO and UNICEF estimates of Immunization Coverage: 2018 revision July 2, 2019.

4. Concern over possible resurgence of vaccine preventable diseases among children The Week, May 24, 2020.

5. Time to address the challenge amid COVID 19 lockdown: Dr Vijay Yewale, ET Health World April 27,2020.

6. Dealing with immunization during the COVID 19 pandemic: India's experience, 26 May 2020.

7. National Immunization Schedule. https://main.mohfw.gov.in.

8. Swift action to prioritize vaccinations for children post COVID-19 lockdown, EH News Bureau, April 21, 2020.

9. Vaccine rates drop dangerously as parents avoid Doctor's visit, New York Times, April 24, 2020.

10. Kiely M, Boulianne N, Talbot $D$, et al. Impact of vaccine delays at the $2,4,6$ and 12 month visits on incomplete vaccination status by 24 months of age in Quebec, Canada. BMC Public Health 2018;18:1364. DOI: 10.1186/s12889-018-6235-6. 